\title{
¿Agujeros de gusano y Viajes en el Tiempo? No es Probable
}

\section{doi: $10.52749 /$ fh.v2i3.10}

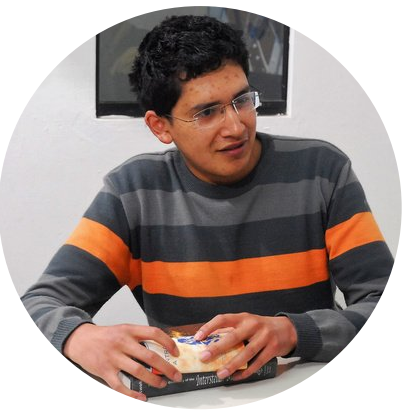

\begin{abstract}
ADRIAN DE LA CRUZ
Miembro de la Sociedad Secular Humanista del Perú (SSH) y del Instituto de Estudios Transhumanistas (IET). Bachiller de Ingeniería Civil de la Universidad Nacional del Centro del Perú, Huancayo - Junín. Es activista escéptico, divulgador de física y miembro de la Asociación Peruana de Astrobiología.
\end{abstract}

elo.d.hdelacruz@gmail.com

Resumen. Los agujeros de gusano han avanzado tanto como un método para eludir las limitaciones de la velocidad de la luz, así como un medio para construir una máquina del tiempo (para viajar al pasado). Así, se argumenta que la Relatividad General puede permitir estas dos posibilidades. En este artículo se argumenta que los agujeros de gusano conectan regiones contrarias desconectadas causalmente, violando dos de los principios más fundamentales de la física, a saber, la conservación de la energía local y el principio de incertidumbre energía-tiempo.

Palabras clave: Agujero negro, agujero de gusano, viaje en el tiempo, máquina del tiempo, leyes físicas

\section{Agujeros de gusano}

Un agujero de gusano es una geometría espacial múltiplemente conexa que consiste en dos "agujeros con boca" separados por una distancia. Los labios de la boca se identifican porque existe un atajo a través del agujero de gusano. Yendo por el camino largo, la distancia entre las bocas, llámese $L$, puede ser enorme e incluso infinita. Por el contrario la distancia I, a través del agujero de gusano, puede ser muy pequeña. Según algunos autores, estos agujeros de gusano podrían ser restos del big bang como también geometrías artificialmente construidas. Los agujeros de gusano son muy comunes, se habla de ellos con frecuencia en la prensa, en las historias de ciencia ficción y en los libros de física para una audiencia masiva.

Tales agujeros de gusano, si es que existen, serían ventanas muy interesantes de regiones distantes del espacio - incluso más allá de nuestro horizonte cósmico. Podríamos pasar por una, echar un vistazo alrededor y volver a informar de lo que vimos (Greene, 2006; Thorne, 2010). Si no fuéramos lo suficientemente valientes para hacer eso, podríamos enviar una cámara de televisión conectada a un cable. Se ha recomendado que los agujeros de gusano se usen como medio para escapar de un moribundo universo-burbuja a uno más joven y seguro (Kaku, 2011). Si existieran, podrían dar un significado operativo a la idea de "Multiverso". Por último, se especula que puedan ser componentes de supuestas máquinas del tiempo.

Al analizar estas afirmaciones, es importante darse cuenta de que los agujeros de gusano transitables (TW's) no son posibles en la física clásica. La Relatividad General clásica no permite a un agujero de gusano, tal como un Puente de Einstein-Rosen, permanecer abierto el tiempo suficiente para que un objeto pase de una boca a otra. La idea en la mecánica cuántica, por lo general en la forma del efecto Casimir, puede permitir violaciones de las condiciones de energía positiva que prohíben los TW's. Demos a los agujeros de gusano el beneficio de la duda y asumamos que los efectos cuánticos pueden estabilizarlos; sin embargo, debido a que no existen en el límite clásico, un análisis coherente debe tener en cuenta las limitaciones de la mecánica cuántica, en particular, el principio de incertidumbre. Eso y el hecho de que la energía es una carga gauge, es todo lo que se utilizará en este trabajo.

Puramente a efectos de poder vizualizar la geometría, analizaremos la cuestión en $2+1$ dimensiones. Comencemos por describir una geo- 
metría espacial de dos dimensiones que contiene un agujero de gusano. Tomamos una hoja grande de papel normal y la doblamos de modo que los puntos distantes estén cerca y conectados en un espacio de tres dimensiones. Luego cortamos dos bocas e identificamos los bordes. Si el camino más largo es muy grande podemos ignorar el hecho de que las dos hojas están conectadas (excepto por el agujero de gusano) y pensamos en ellos como dos universos separados conectados por un agujero de gusano. Llamamos a las dos hojas A y B. Vivimos en A.

Visto desde cualquier lado, los agujeros de gusano pueden tener masa, motivo por el que crean un campo gravitatorio que en $2+1$ dimensiones significa un déficit cónico. En ese caso, la geometría sería un par de conos conectados en sus puntos por una región suavizada de curvatura negativa. Dicho sea de paso, el hecho de que el agujero de gusano está curvado negativamente es una manifestación explícita de que se necesita energía negativa para apoyar la configuración.

\section{El caso eléctrico}

Vamos a empezar un experimento simple que involucra a dos cajas (cajas 1 y 2) que pueden contener partículas cargadas eléctricamente. Llame a las partículas mesones $\pi^{+}$. Podemos construir un estado (trabajamos en un gauge de Coulomb) con una carga total $\mathrm{N}$ y una fase relativa $\theta$.

$$
|N \theta\rangle=\sum_{n=0}^{N}|N-n, n\rangle e^{i n \theta}
$$

donde $|\mathbf{m}, \mathbf{n}\rangle$ significa un estado con carga y en la caja 1 y $\mathbf{2}$. La fase relativa es mensurable y se puede medir de la siguiente manera. Un haz de neutrones se divide en dos haces. Un haz se envía a la caja 1 y el ntro a la caja 2. Al pasar por la caja de mesones $\pi^{+}$,una carga puede ser absorbida, convirtiendo un neutrón en un protón. Cuando se vuelven a combinar los haces, la probabilidad de encontrar un protón contiene un término de interferencia proporcional a $\cos 2 \theta$.

Típicamente, la fase no será independiente del tiempo, pero debido a la energía electrostática de Coulomb, oscilará de una manera regular y predecible. Las cajas se pueden separar con las relaciones de fase restante intacta.
Imagina que las dos cajas empiezan a nuestro lado del agujero de gusano en la hoja A. Ahora transportamos la caja 2 al otro extremo del agujero de gusano, tomando el camino más largo. Al final de este proceso tenemos dos depósitos de carga, uno en cada hoja, con la fase relativa definida, lo que nos proporciona un medio para comparar fases en las dos bocas.

A continuación, pasamos la caja $\mathbf{1}$ a través del agujero de gusano para que aparezca en la hoja $\mathbf{B}$. Ahora ambas cajas se encuentran en la misma hoja y presumiblemente tienen una fase definitiva que se puede comparar. Pero, de hecho, cuando comparamos experimentalmente las fases nos encontramos con que están totalmente no correlacionados: el término de interferencia proporcional a $\cos \theta$ está ausente.

Para ver por qué es así, vamos a examinar más de cerca lo que ocurre cuando una carga se pasa a través de un agujero de gusano. Cuando la carga comenzó en la hoja A, las líneas de flujo eléctrico irradiaban hacia el infinito en este lado. Al pasar la carga a través del agujero de gusano, las líneas de flujo, inesperadamente, no se reordenan asintóticamente. Ellos logran regresar a través del agujero de gusano y continúan radiando hasta el infinito en la hoja A. En otras palabra, pasa SIN CARGA a través del agujero de gusano. En realidad el observador en el lado B ve que además de la carga directa, una carga opuesta se ha formado en la boca del agujero de gusano. En el lado A la carga también se ha modificado, pero ahora existe en la forma de una boca de agujero de gusano cargada.

Ahora vamos a volver al problema de las dos cajas cargadas con fases relativas definidas. Después de enviar la caja 1 a través del agujero de gusano, hay cuatro sistemas encargados de hacer un seguimiento de las dos cajas y de las dos bocas del agujero de gusano, A y B. En lugar de encontrar las fases de las dos cajas correlacionadas, lo que se encuentra es una correlación de fase entre la caja $\mathbf{2}$ y la boca A, y una correlación similar entre las fases de $\mathbf{1}$ y B, pero no hay correlación entre las fases $\mathbf{1}$ y $\mathbf{2}$.

\section{El tiempo y la transferencia de energía}

Energía y tiempo están relacionados de la misma manera como carga y fase. Vamos a repetir el análisis y ver lo que dice en este caso. Para facilitar la visualización del sistema, trabajamos en 2+1 dimensiones, pero el análisis se aplica en dimensiones más altas. 
Primero considere lo que pasa cuando una masa pasa a través del agujero de gusano desde $\mathbf{A}$ a $\mathbf{B}$. Por simplicidad considere una masa puntual. El análogo de las líneas de flujo eléctrico es el déficit cónico que se extiende hasta el infinito en la hoja A. Al igual que en el caso eléctrico, cuando la masa pasa a través del agujero de gusano, el déficit cónico permanece detrás y ningún cambio se lleva a cabo en cualquiera de los lados. En el lado $\mathbf{A}$ el déficit parece ser debido a la boca $\mathbf{A}$. En el lado $\mathbf{B}$ el déficit es compartido entre la masa puntual y la boca B, que parece haber perdido toda la masa aportada por la masa puntual que expulsó.

Vamos a empezar de nuevo. En vez de dos cajas de carga que sirven como fases confiables, tomamos una energía dada y repartimos dentro dos relojes, llamados $\mathbf{1}$ y $\mathbf{2}$. Las variables de fase se sustituyen por las lecturas de tiempo $t_{1}$ y $t_{2}$. Los relojes se pueden preparar inicialmente de modo que la diferencia de tiempo, $t_{1}-t_{2}$ sea cero: en otras palabras los relojes están sincronizados.

Luego transportamos el reloj $\mathbf{2}$ a lo largo del camino hacia la hoja B. Si esto se hace sin gran velocidad y sin pasar a través de pozos potenciales gravitacionales, los dos relojes permanecerán sincronizados. En cualquier caso no hay ninguna dificultad en la contabilización de cualquier diferencia en los tiempos propios a lo largo de los respectivos caminos de los relojes. Ahora tenemos relojes que pueden compararse cerca de las dos bocas.

Este es el experimento que nos gustaría hacer. Pasar el reloj $\mathbf{1}$ a través del agujero de gusano y compararlo con el reloj 2. Después de anotar el resultado, el reloj $\mathbf{1}$ puede volver a través del agujero de gusano y reportar su hallazgo.

Uno podría esperar ingenuamente que, aparte de los efectos que podrían ocurrir en el agujero de gusano, los dos relojes deberían coincidir. En otras palabras el tiempo relativo observable $\left(t_{B}-t_{A}\right)$ se espera que sea muy pequeño en comparacıon con L. Pero está claro que esto no puede ser. En cambio los dos relojes estarán completamente no correlacionados. Esto es una consecuencia del principio de incertidumbre energía-tiempo y el hecho de que la energía que pasa a través del agujero de gusano era exactamente igual a cero. Por lo tanto podemos incluir que

$$
\left\langle\left(t_{B}-t_{A}\right)^{2}\right\rangle=\infty
$$

¡Tenga en cuenta que el reloj $\mathbf{1}$ tiene la misma probabilidad de salir en el pasado infinitamente remoto como en el futuro infinitamente remoto!

Otra forma de decir lo que sucedió es que cuando el reloj $\mathbf{1}$ entró y salió en $\mathbf{A}$ y $\mathbf{B}$, dejó atrás los relojes en las bocas del agujero de gusano. Después de salir en el $\mathbf{B}$, el reloj $\mathbf{1}$ se encontraba sincronizado con el reloj en la boca $B$, pero totalmente al azar con respecto al reloj 2.

Hay un parámetro adicional que se necesita para especificar un agujero de gusano, es decir, una variable de tiempo relativo que especifica cómo se realiza la identificación. Por ejemplo, dos agujeros de gusano estacionarios serán identificados en el mismo tiempo en el marco de reposo o no podrá ser compensado por un cierto retraso finito o adelantado. Obviamente si el retraso es finito esto no hará diferencia alguna para el argumento, aunque el tiempo de retardo es algo incierto, mecanocuánticamente.

Una manera de fijar el tiempo de retraso es comenzar con un agujero de gusano en el que es tan pequeña la "forma larga" y a través del agujero de gusano. Algunos autores han especulado que estos pequeños agujeros de gusano podrían ser creados en el laboratorio. Una vez creados, se pueden separar lentamente a una gran distancia. Si se separan simétricamente, el tiempo de retardo debe ser cero por simetría. Por otra parte, no hay ninguna razón de por qué la demora fluctuaría más allá de cualquier pequeña fluctuación de no haber estado allí desde el comienzo.

\section{El argumento}

Comencemos por examinar mi argumento. Empecemos con el caso de la carga eléctrica. Comenzamos con la suposición de un agujero de gusano con dos agujeros de boca muy distantes, A y B. Además, el sistema contiene dos cajas de carga con una carga total $N$. Las cajas se han preparado con una fase relativa definida entre ellas,

$$
|N \theta\rangle=\sum_{n=0}^{N}|N-n, n\rangle e^{i n \theta}
$$

Por simplicidad tomamos $\theta=0$.

Las dos cajas se localizan inicialmente cerca de las dos bocas del agujero de gusano, caja $\mathbf{1}$ cerca de $\mathbf{A}$ y caja $\mathbf{2}$ cerca de $\mathbf{B}$. El experimento consiste en mandar la caja $\mathbf{1}$ a través del agujero de gusano y 
posteriormente medir la relación de fase entre las cajas cuando están en el lado B.

Existen tres grados de libertad relevantes en el problema. Los dos primeros son las cargas de las cajas o equivalentemente, las fases conjugadas, $\theta_{1}$ y $\theta_{2}$. El tercer grado de libertad es el valor entero del flujo eléctrico a través del agujero de gusano, llamado $f$. El flujo es la variable conjugada para la fase del lazo de Wilson, asociado con el ciclo no trivial que pasa a través del agujero de gusano, y

a continuación, regresa por el camino largo. Llámese a ese ángulo $\omega$.

Arriba se asumió que al inicio del experimento el flujo desapareció. Que es equivalente a decir que las bocas del agujero de gusano son eléctricamente neutras. Suponiendo que una carga pasa a través del agujero de gusano desde A a B. El resultado será que el flujo se excita de modo que $f=n$. En efecto, el agujero de gusano ha "medido" la carga de la caja 1. Más precisamente, la carga de la caja ha conseguido enlazarse con el grado de libertad del flujo. Esto, obviamente, tiene el efecto de decoherencia de la relación de fase entre 1 y $\mathbf{2}$. El experimento también se puede describir en base a la fase. Inicialmente la función de onda de las tres variables relevantes es

$$
\psi\left(\theta_{1}, \theta_{2}, \omega\right)=\delta\left(\theta_{1}-\theta_{2}\right)
$$

la función de onda es independiente de $\omega$ porque el flujo es cero.

Después que la caja 1 pase a través del agujero de gusano, la función de onda se convierte en

$$
\psi\left(\theta_{1}, \theta_{2}, \omega\right)=\delta\left(\theta_{1}-\theta_{2}-\omega\right)
$$

Obviamente la probabilidad para valores dados de $\theta_{1}-\theta_{2}$ es perfectamente plano si no se mide. La relación de fase relativa ha sido retirada según lo anterior.

Pero ahora vamos a empezar con un estado inicial diferente para el agujero de gusano. En lugar de lo que es un vector propio de flujo, y por tanto de las cargas de la boca, tomemos un vector propio de la fase del lazo de Wilson $\omega$, con valor propio $\omega_{0}$. Por lo tanto, el estado inicial antes que $\mathbf{1}$ pase a través del estado del agujero de gusano es

$$
\psi\left(\theta_{1}, \theta_{2}, \omega\right)=\delta\left(\theta_{1}-\theta_{2}\right) \delta\left(\omega-\omega_{0}\right)
$$

Ahora pasa la caja 1 hasta $B$. El resultado es fácil de operar y la función de onda final se ve fácilmente que

$$
\psi\left(\theta_{1}, \theta_{2}, \omega\right)=\delta\left(\theta_{1}-\theta_{2}-\omega_{0}\right) \delta\left(\omega-\omega_{0}\right)
$$

En otras palabras, la diferencia de fase entre las cajas se ha desplazado por la constante $\omega_{0}$, pero se mantiene coherente. Evidentemente mi demanda (que la relación de fase ha sido retirada) es contingente sobre la suposición de que la boca del agujero de gusano comienza en estados propios de carga. Un análisis más general haría indicar que si el flujo inicial (cargas de la boca) tiene la incertidumbre $\delta f$, entonces las diferencias de fase de las dos cajas pueden medirse hasta la precisión 1/ $(\delta f)$.

Puede parecer extraño situar las cargas de boca iniciales en una superposición coherente de estados. Hay una razón por la cual es difícil de crear y observar los estados de este tipo en la física ordinaria. El punto es que hay un precio de la energía a pagar por un flujo $f$ que es proporcional a $f^{2}$. Esto proporciona una energía cinética para la fase $\omega$. Esto implica que los elementos de la diagonal (en la $f$ base) varían rápidamente con el tiempo y son ordinariamente un promedio de más. Pero, nos permitimos pasar por alto tales efectos para las cargas y para las cajas, así que no vemos ninguna razón para no hacerlo para las bocas de los agujeros de gusano.

Pasemos ahora al caso de interés real, en que la energía y el tiempo reemplazan a la carga y la fase. En el análisis, se asume que las bocas del agujero de gusano comienzan con energía infinitamente precisa, i.e., que los ángulos con déficit (o energía $A D M$ en $D>3$ ) de cada boca son demasiado altos. Este es el sistema operativo análogo a partir de un fuerte flujo del agujero de gusano en lugar de la fase aguda del agujero de gusano. Las preguntas obvias que cualquier físico competente hubiera hecho son: ¿cuál es el significado de la fase correspondiente en el problema de energía-tiempo?, y ¿es probable que se defina nítidamente?

El análogo del lazo de Wilson tiene que ver con la ambigüedad en cómo se identifican las bocas del agujero de gusano $\omega_{0}$. La más obvia y simple identificación geométrica es identificar a ellos en tiempos iguales en el sistema en reposo. Pero también es posible identificar con un desplazamiento del tiempo $\boldsymbol{\tau}$. Esta variable es muy similar a la fase del lazo de Wilson y se corresponde con el cambio de hora de una trayectoria espacial 
semejante como la que pasa por el agujero de gusano y regresa por el camino largo. Es completamente obvio que si las bocas del agujero de gusano tienen energía infinitamente precisa, a continuación, $\boldsymbol{\tau}$ es totalmente indefinido. El resultado en este caso es, como se informó aquí, que el reloj $\mathbf{1}$ y el reloj $\mathbf{2}$ están completamente al azar el uno con el otro, al final del experimento.

¿Pero $\tau$ es razonablemente aleatorio y es infinitamente intensa la energía de la boca? La respuesta depende en cómo se hizo el agujero de gusano. Supongamos que se ha hecho a nivel local por un proceso que crea las dos bocas cerca una de la otra, a un tiempo más o menos bien definido. Obviamente $\tau$ empieza pequeño, quizá con alguna modesta incertidumbre. La masa total del sistema puede ser definida, pero la diferencia de masa (conjugado para $\tau$ ) está limitada por el principio de incertidumbre y debe ser incierta. Así, la situación sería análoga a la del experimento con una más o menos bien definida fase de Wilson. En este caso los relojes no llegarían al azar mediante el paso a través del agujero de gusano.

Una cuestión interesante surge si consideramos la analogía de la energía electrostática o las cargas de la boca $\left(f^{2}\right)$ que desempeñaron el papel de un término cinético para la fase de Wilson. La energía electrostática es cuádrica en la carga, pero la energía es lineal en la masa. La energía de los dos agujeros de gusano es $M_{1}+M_{2}$. Esta es completamente independiente de la diferencia de masa y por lo tanto no juega el papel de un término cinético de $\boldsymbol{\tau}$. No hay tendencia a llevar fuera los elementos de la diagonal entre diferentes masas de agujeros de gusano y no hay tendencia a extenderse la distribución de probabilidad para $\boldsymbol{\tau}$.

Esto significa que los agujeros de gusano no tienen sentido.

\section{Conclusión}

Los agujeros de gusano que permitan a un observador un breve circuito por grandes distancias espaciales introduciéndose por una boca provocan otra violacíon de la mecánica cuántica. La magnitud medida de tiempo entre la entrada y la salida es infinito como consecuencia del principio de incertidumbre y el hecho de que la transferencia de energía total ADM debe ser exactamente igual a cero.

La única interpretación razonable es que los acontecimientos de las dos bocas del agujero de gusano están completamente no correlacionadas. La apariencia de un reloj similar al reloj $\mathbf{1}$ en la hoja B se puede interpretar como una emisión cuántica por un objeto inestable, a saber, la boca B. Este decaimiento puede tener lugar en cualquier momento y no está correlacionado con el evento de "absorción" en A. Creo que incluso está justificado decir que el observador no se apartaba de A. Se dejó atrás en la forma de los niveles de energía complejos de la boca A.

En cuanto a los viajeros, tendrán que buscar otro tipo de máquina del tiempo.

\section{Referencias}

Greene, B. (2006). El tejido del cosmos. Crítica.

Kaku, M. (2011). Universos paralelos. Atalanta.

Thorne, K. S. (2010). Agujeron negros y tiempo curvo. Crítica.

\section{Cómo citar este artículo:}

De La Cruz, A. (2021). ¿Agujeros de gusano y viajes en el tiempo? No es probable. Futuro Hoy, 2(3), 55-59. https://doi.org/10.52749/fh.v2i3.10

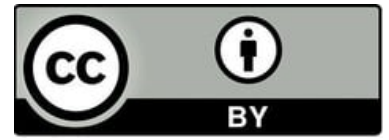

Esta obra está bajo licencia internacional Creative Commons 4.0 Reconocimiento 4.0. 